\title{
ESTUDIO EXPERIMENTAL DE UNA FORMA DE EDUCACION PRENATAL
}

\author{
CLINICA DE MATERNIDAD "DAVID RESTREPO"
}

Doctores Alberto Cárdenas Escovar, Eduardo Acosta Lleras, Kafael Zornosa del Valle y Carlos Alvear Machado

I. Teoría de las bases neuro-fisiológicas del método psicoprofiláctico para el parto sin dolor.

"Las raíces de este método se encuentran en los principios del antiguo método hipno-sugestivo, basado en las doctrinas de Pavlov" ha dicho Nicolaiev.

En efecto, el Método Psicoprofiláctico ha sido el resultado de la evolución de los métodos hipno-sugestivos aplicados a la analgesia obstétrica, y la fisiología reflexológica pavloviana ha contribuído a esta transformación, suministrando al nuevo método los elementos necesarios para pasar del empirismo a la sistematización científica.

La teoría de la psicoprofilaxia es muy conocida y resultaría innecesario y prolijo extendernos sobre ella. Además de las publicaciones iniciales de los autores rusos Platonov, Velvovski, Plotitcher, Chougom, Nicolaiev y otros, disponemos de los trabajos franceses, entre los cuales los fundamentales se deben a
Fernand Lamaze y a sus colaboradores, Vellay, Hersilie, Angelergues, Bourrel y Vermorel, que cubren el período comprendido entre 1952 y 1956 . Nos limitaremos aquí a enumerar sucintamente los principios reflexológicos que sirven de base a la fisiología del método psicoprofiláctico.

1. En primer lugar, recordemos que los autores del método insistieron inicialmente sobre la psicogénesis del dolor, negando que existiera en el parto normal una excitación directa de los receptores del dolor en el tractus genital, pero ulteriormente modificaron esta opinión y admitieron un substratum fisiológico del fenómeno doloroso, representado por las interocepciones originadas en aquellos receptores: estiramientos y desalojamientos del útero, de sus ligamentos y vasos, contracción uterina, presión de la presentación sobre el segmento inferior y el cuello uterinos, vasoconstricción o vasodilatación de los vasos uterinos, modificaciones del qui- 
mismo tisular resultantes de la contracción, etc.

La vía centrípeta de conducción de estos estímulos está representada por fibras sensibles del simpático, que por los rami-comunicantes y las raíces anteriores alcanzan las células medulares simpáticas correspondientes (primera neurona); las fibras que luégo se cruzan y se unen al haz espino-talámico lateral (segunda neurona), y finalmente una tercera neurona tálamo-cortical.

Ahora bien, la elaboración necesaria para que las interocepciones uterinas, pertenecientes dentro de la terminología reflexológica al "primer sistema de señalización", se hagan dolorosas, es explicada como veremos a continuación.

2. El dolor, con todas sus manifestaciones subjetivas y objetivas, se considera como un reflejo incondicionado; pero en el caso particular del parto normal, el dolor es un reflejo condicionado cuya presencia en la mujer embarazada se explica por la creación de conexiones transitorias corticales entre los centros o focos de manifestación dolorosa por una parte, y los focos de excitación propios de las interocepciones uterinas por otra, gracias a la acción repetida de la palabra "segundo sistema de señalización", que por un error tradicional de la educación, asocia fatalmente los conceptos de "dolor" y "parto", de manera que la contracción uterina viene a ser la señal del dolor, y este reflejo condicionado se mantiene y refuerza en la mujer desde la adolescencia, agravándose por la ignorancia y el ambiente de falsos temores que rodean a la mujer joven, la embarazada y la parturienta. Esta acti- vidad reflejo-condicionada está regida por la inducción constante de dos procesos antagonistas, la excitación y la inhibición, que regulan el grado de difusión de los focos de actividad en la corteza, así como las relaciones córticosubcorticales. A la dinámica de estos procesos se ha llamado "actividad nerviosa superior", y ella es el objeto de los esfuerzos educativos en el método psicoprofiláctico.

3. La finalidad del método es reorganizar la actividad nerviosa superior, reemplazando los reflejos condicionados dolorosos por reflejos condicionados útiles susceptibles de asociar la contracción uterina del parto a cierta actividad positiva de la corteza cerebral, que induzca negativamente una limitación de las interocepciones pélvicas. Tal resultado es el "freinage" de la terminología francesa.

La substitución de reflejos condicionados para el parto sin dolor se logra principalmente por medio del lenguaje, cuya importancia como segundo sistema de señalización ya vimos, y que permite suprimir los prejuicios y la ignorancia, crear alrededor de la mujer un ambiente propicio para la maternidad, impartirle una educación sobre la fisiología del embarazo y del parto, y completar o mantener una actividad cortical positiva durante todo el trabajo y el nacimiento. Esta disposición se refuerza eficazmente gracias a un entrenamiento físico especial consistente en varios tipos de ritmo respiratorio aplicables en las diversas etapas del trabajo, y en el dominio de la relajación muscular, todo lo cual debe aprenderse y practicarse prenatalmente, en forma 
que desde la iniciación de los mecanismos del parto la contracción uterina actúe como señal desencadenante de esta actividad refleja benéfica, y por ende de la actividad cortical indolorizante.

\section{Material y métodos.}

Este estudio comprende 1.543 casos obstétricos atendidos en la Clínica de Maternidad David Restrepo de Bogotá, por el Método Psicoprofiláctico, en el período comprendido entre febrero de 1959 y marzo de 1962.

Esta es una institución destinada a la atención prenatal y obstétrica de pacientes pertenecientes a la llamada "clase media económica" de la ciudad.

Los casos estudiados pueden considerarse homogéneos desde los puntos de vista socio-económico y cultural. Igualmente homogéneos son, por fuerza, los métodos de entrenamiento y de atención obstétrica empleados por el personal médico y de preparadoras, quienes han adquirido a su vez un adiestramiento uniforme para la psicoprofilaxia en la misma clínica.

La práctica de la psicoprofilaxia en la Clínica Restrepo está basada en el llamado Método Psicoprofiláctico para el parto sin dolor, cuyo origen corresponde a los obstetras y neurofisiólogos rusos, seguidores de la escuela reflexológica de Pavlov, y cuya difusión en el mundo occidental se debe al doctor Fernand Lamaze de París. Hemos elegido desde el comienzo de nuestra experiencia este método con exclusión de otros, con el ánimo de cumplir una etapa experimental cuya metodología uniforme nos permitiera, al alcanzar una casuís- tica suficientemente voluminosa, formar un juicio claro sobre el valor de este tipo de labor educativa y de atención obstétrica. Queremos valorar, objetivamente y sin prejuicio, una forma particular de psicoprofilaxia y juzgar la conveniencia de cortinuar su aplicación, o de modificarla, para beneficio de la embarazada, tanto desde el punto de vista obstétrico como en los aspectos educativos, familiares y sociales.

Sería inútil describir en forma detallada la teoría y la práctica del Método Psicoprofiláctico, pues ellas se encuentran en numerosas publicaciones tales como las que aparecen en nuestra lista bibliográfica.

\section{Preparación y atención obstétrica.}

La preparación se efectúa en pacientes inscritas voluntariamente en los cursos de psicoprofilaxia. Se comienza el entrenamiento alrededor de las 32 semanas de gestación y no se emplea discriminación alguna, de modo que solamente quedan excluídas aquellas que declaran no estar interesadas en el método, o hallarse imposibilitadas para concurrir regularmente a las clases por dificultades domésticas. Cada curso dura cuatro semanas e incluye un promedio de 130 pacientes.

La preparación comprende cuatro clases teóricas realizadas por los Médicos ante grupos de 25 a 30 pacientes, acompañadas por sus maridos:

Primera clase: Generalidades sobre el método.

Segunda clase: Anatomía y fisiología de los órganos genitales. Evolución del embarazo. Higiene prenatal. 
Tercera clase: Estudio de los períodos del trabajo y del comportamiento de la paciente.

Cuarta clase: Bases elementales de neurofisiología y explicación de la actividad nerviosa superior.

Las clases prácticas son realizadas por Preparadoras y comienzan después de la primera clase teórica. Las pacientes concurren en grupos de 12 a 15.

Primera clase: Entrenamiento de relajación muscular.

Segunda clase: Ejercicios respiratorios. Diferentes tipos de respiración aplicables en los diversos períodos del trabajo.

Tercera clase: Entrenamiento para los esfuerzos expulsivos.

Cuarta clase: Revisión. Visita a las salas de trabajo y de parto.

Desde el ingreso de la paciente en trabajo, es colocada en una sala de trabajo individual bajo la vigilancia directa y continua de la preparadora de turno, y, desde luego, sometida a los exámenes obstétricos inicial y de control, a cargo de los médicos.

Para el período de expulsión es trasladada a la sala de partos, siempre acompañada por la preparadora, pero el médico obstetra asume entonces la dirección del comportamiento de la paciente y la atención del nacimiento.

\section{Medicación.}

Al valorar los resultados de un caso de psicoprofilaxia, con frecuencia se presenta la dificultad de establecer hasta qué grado el empleo de algunos me- dicamentos en el trabajo puede desvirtuar el concepto sobre la acción indolorizante atribuída al método. Debemos advertir, en primer lugar, que las finalidades que perseguimos son la correcta atención obstétrica, la protección de la madre y del niño, y el alivio de la madre, sea por la psicoprofilaxia o por los métodos usuales. En otras palabras, la preparación psicoprofiláctica de la paciente no nos obliga a la abstención de los medicamentos análgésicos una vez que observemos el fracaso de aquélla, cinéndonos, desde luego, a las normas de la correcta analgesia medicamentosa. Así lo advertimos previamente a las pacientes, y no vacilamos en la práctica de esta política. Pero nos ha interesado establecer criterios justos para el estudio de estos casos de "analgesia mixta", por la necesidad de comparar nuestros resultados con los de otros centros y de valorar lo más objetivamente posible los resultados del método psicoprofiláctico.

Por lo que respecta a la controversia sobre el uso de los agentes propiamente analgésicos, nuestro criterio se expresa diciendo que si la indicación primaria del medicamento en cuestión sirve para prevenir o corregir ciertos tipos de distocia susceptible de causar la aparición del dolor, no se puede, entonces, hablar de que se ha usado un "analgésico", y en ese caso la aplicación de la droga es legítima y necesaria, no sólo desde el punto de vista obstétrico, sino también dentro de la metodología de la psicoprofilaxia bien dirigida. El ejemplo clásico de este caso es el uso de la Meperidina administrada para corregir una incoordinación uterina, antes 
de que ésta llegue a producir dolor en una paciente que hasta el momento está recibiendo el beneficio completo de su preparación psicoprofiláctica.

\section{Calificación:}

Nos ha parecido conveniente simplificar el sistema de calificación de los casos, tratando de hacerlo más objetivo, $\mathrm{y}$ en consecuencia hemos empleado el que describiremos a continuación, que fue propuesto por nosotros y aceptado en el II Simposio Colombiano de Psicoprofilaxia Obstétrica, reunido en Medellín en 1960. Este sistema consta solamente de tres grupos así:

Caso Bueno: Categoría que engloba todos los casos en que no hay dolor verdadero en ningún período del trabajo.
Caso Regular: Aquel en que no fue posible suprimir realmente el dolor, pero en el que se obtuvo un beneficio innegable expresado en el comportamiento, la colaboración y la satisfacción de la paciente.

Caso Malo: Caracterizado por dolor, excitación psico-motriz y falta de colaboración, lo mismo que en las pacientes no preparadas.

\section{Resultados.}

Los resultados obtenidos en los 1.543 casos obstétricos objeto de este estudio, se presentan en los cuadros siguientes, en los cuales los casos han sido distribuídos según la paridad, el tipo del nacimiento y la calificación.

CUADRO 1

NUMERO DE CASOS ATENDIDOS POR EL METODO

\begin{tabular}{|c|c|c|c|c|c|c|}
\hline Primigestantes & 467 & $=$ & $30,26 \%$ & sobre & 1.543 & casos \\
\hline Multigestantes & 1.076 & $=$ & $69,74 \%$ & sobre & 1.543 & casos \\
\hline TOTAL & 1.543 & & & & & \\
\hline
\end{tabular}

CUADRO 2

PARTOS ESPONTANEOS ATENDIDOS POR EL METODO

\begin{tabular}{lrlll}
\hline Primigestantes & 370 & $=26,50 \%$ & sobre & 1.396 \\
Multigestantes & 1.026 & $=73,48 \%$ & sobre & 1.396 \\
& & & & \\
TOTAL & 1.396 & $90,47 \%$ & sobre & 1.543 \\
\hline
\end{tabular}

CUADRO 3

APLICACIONES DE FORCEPS EN PACIENTES PREPARADAS

\begin{tabular}{lrllll}
\hline Primigestantes & 84 & $=$ & $66,66 \%$ & sobre & 126 \\
Multigestantes & 42 & $=$ & $33,33 \%$ & sobre & 126 \\
& & & & & \\
TOTAL & 126 & & $8,16 \%$ & sobre & 1.543 \\
\hline
\end{tabular}


CUADRO 4

OPERACIONES CESAREAS EN PACIENTES PREPARADAS

\begin{tabular}{lrlll}
\hline Primigestantes & 13 & $=61,90 \%$ & sobre & 21 \\
Multigestantes & 8 & $=38,09 \%$ & sobre & 21 \\
& 21 & & & \\
TOTAL & $2,36 \%$ & sobre & 1.543 \\
\hline
\end{tabular}

Los casos fueron calificados según el sistema de tres clases ya explicado, y los resultados fueron los siguientes:

CUADRO 5

\begin{tabular}{lrcrc}
\hline \multicolumn{2}{l}{ Casos en Primigestantes } & Buenos & Regulares & \multicolumn{1}{c}{ Malos } \\
\hline No operatorios & $\mathbf{3 6 6}$ & $159(43,42 \%)$ & $77(21,04 \%)$ & $130(35,52 \%)$ \\
Operatorios & $\mathbf{1 0 1}$ & $39(38,71 \%)$ & $25(24,75 \%)$ & $37(36,53 \%)$ \\
& $\mathbf{4 6 7}$ & $198(42,48 \%)$ & $102(21,84 \%)$ & $167(35,67 \%)$ \\
TOTALES & & Buenos & Regulares & Malos \\
Casos en Multigestantes & $\mathbf{1 . 0 2 6}$ & $499(48,63 \%)$ & $296(28,85 \%)$ & $231(22,51 \%)$ \\
No operatorios & 50 & $12(24,00 \%)$ & $14(28,00 \%)$ & $24(48,00 \%)$ \\
Operatorios & $\mathbf{1 . 0 7 6}$ & $511(47,58 \%)$ & $310(28,71 \%)$ & $255(23,69 \%)$ \\
TOTALES & & & &
\end{tabular}

CUADRO 6

\begin{tabular}{lrrrc}
\hline \multicolumn{1}{l}{ Aplicaciones de fórceps } & \multicolumn{1}{c}{ Buenos } & Regulares & Malos \\
\hline Primigestantes & $\mathbf{8 4}$ & $30(35,72 \%)$ & $20(23,80 \%)$ & $34(40,47 \%)$ \\
Multigestantes & $\mathbf{4 2}$ & $9(21,42 \%)$ & $13(30,95 \%)$ & $20(47,28 \%)$ \\
& $\mathbf{1 2 6}$ & $39(30,95 \%)$ & $33(26,19 \%)$ & $54(42,85 \%)$ \\
TOTALES & & & \\
\hline
\end{tabular}

CUADRO 7

\begin{tabular}{lrccc}
\hline Operaciones cesáreas & & Buenos & Regulares & Malos \\
\hline $\begin{array}{l}\text { Primigestantes } \\
\text { Multigestantes }\end{array}$ & $\mathbf{1 3}$ & $3(23,07 \%)$ & $3(23,07 \%)$ & $7(53,84 \%)$ \\
TOTALES & $\mathbf{8}$ & $5(62,50 \%)$ & $2(25,00 \%)$ & $1(12,50 \%)$ \\
& $\mathbf{2 1}$ & $8(38,09 \%)$ & $5(23,81 \%)$ & $8(38,09 \%)$ \\
\hline
\end{tabular}

CUADRO 8

\begin{tabular}{lrrc}
\hline Resultados globales & Buenos & Regulares & Malos \\
\hline & $709(45,92 \%)$ & $412(26,70 \%)$ & $422(27,35 \%)$ \\
TOTAL & $1.543(99,97 \%)$ & & \\
\hline
\end{tabular}




\section{CONCLUSIONES}

1. El Método Psicoprofiláctico ha sido aplicado en la Clínica Restrepo de Bogotá durante los pasados tres años, como un ensayo de educación destinado a complementar la atención prenatal y obstétrica.

2. Los resultados obtenidos desde el punto de vista del comportamiento, de la comodidad, de la satisfacción de las pacientes atendidas por este Método y de la supresión real del dolor en un $45,9 \%$ de ellas, justifican la continuación de su empleo y los esfuerzos por mejorar las técnicas de su aplicación.

3. El Método se ha mostrado también como un auxiliar valioso en el progreso de los sistemas educativos médicos en la especialidad, por cuanto su correcta apli- cación demanda un estudio especialmente cuidadoso del caso obstétrico como tal, un ajuste de la conducta a los criterios y normas actuales de la ciencia médica en general, y de la Obstetricia en particular, y un esfuerzo hacia la concepción psico-somática ante los problemas de la embarazada y de la paciente en trabajo de parto.

4. En el grupo estudiado, la frecuencia de intervenciones (fórceps y cesáreas) no muestra diferencias significativas en relación con las ratas usuales en las pacientes de la Clínica no preparadas por el Método.

El valor bajo del índice de cesáreas se explica porque las pacientes con antecedentes de cesárea no suelen tomar el curso de psicoprofilaxia, lo cual excluye de nuestra serie las cesáreas iterativas.

1. Alviar, J. C.: La Aplicación del Método Psicoprofiláctico y sus Resultados en la Clínica David Restrepo. Tesis de Grado, Pontificia Universidad Católica Javeriana, Bogotá, 1960.

2. CARDEnAS, A., ACOSta, E. y ALVIAR, J. C.: Resultados obtenidos en la Clínica David Restrepo con el Método Psicoprofiláctico. Rev. Clín. Mat. Dav. Restr., 1:44, 1960, y Bull. Soc. Intern. Psycho-prophyl. Obstétr., 3:97, 1961.

3. CARDEnAS, A., CAMPOS, H. y Alviar, J. C.: Aplicación del Método Psicoprofiláctico en la Clínica David Restrepo. Revista Colombiana de Obst. y Ginecol., X: 367, 1959, y Bull. Soc. Inter. Psycho-prophyl. Obstétr., 2:67, 1960.

4. CHERTOK, L.: Les Méthodes Psychosomatiques d'Accouchement sans Douleur. L'Expansion Scientifique Française, París, 1957.

5. LAmAze, F.: L'Accouchement sans Douleur. Revue de la Nouvelle Médecine, 7:61, 1956.

6. LAMAZE, F.: Qu'est-ce que l'Accouchement sans Douleur? Ed. Savoir et Connaître, Paris, 1956.

7. Nicolaiev, A.: El Parto sin Dolor. Ed. Cartago, Buenos Aires, 1957.

8. VERMOREL, H.: L'Accouchement sans Douleur. Camugli, Editeur, Lyon. 
9. Equipos de la CLINICA DAVID RESTREPo de Bogotá e IVAN PAVLOV de Bucaramanga: Método simplificado de condicionamiento en psicoprofilaxia obstétrica. Ponencia en el III Simposio Colombiano de Psicoprofilaxia, Bucaramanga, julio de 1961.

10. MATERNITE de la polyclinique PIERRE ROUQUES: L'Accouchement sans Douleur. Revue de Ia Nouvelle Médecine, 3:9, 1954.

11. SOCIEDAD COLOMBIANA DE PSICOPROFIlAXIA OBSTETRICA. Medicación en Psicoprofilaxia Obstétrica. Trabajo presentado al II Simposio Colombiano, Medellín, mayo de 1960. Rev. Clín. Mat. Dav. Restr., 1:34, 1960. 